PESQUIMAT, Revista de la Fac.CC.MM. de la

UNIVERSIDAD NACIONAL MAYOR DE SAN MARCOS

Vol.IV, $N^{\circ} 2$, pag. 55-65, LIMA-PERÚ. Diciembre 2001

\title{
EL ÍNDICE DE INTERSECCIÓN A LO LARGO DE UN CONJUNTO DE TANGENCIA
}

\author{
Renato Benazic Tomé1
}

\begin{abstract}
En el presente trabajo, consideraremos campos vectoriales holomorfos con singularidad aislada dicrítica, cuyos transformados estrictos no presentan singularidades en el divisor. En estas condiciones, es posible asociar a este campo una hipersuperficie de tangencia $S$ y a cada hoja del transformado estricto, un índice de intersección con el divisor. El resultado principal del presente artículo es que dicho índice de intersección es constante en casi todo punto de cada componente irreducible de $S$.
\end{abstract}

\section{INTRODUCCIÓN}

Sea $\mathcal{M}^{n}$ una variedad analítica compleja de dimensión $n$ y consideremos en ella una foliación analítica singular por curvas. Esto significa que en cualquier punto $p \in \mathcal{M}^{n}$ la foliación es generada por el campo vectorial holomorfo

$$
Z=\sum_{i=1}^{n} Z_{i} \frac{\partial}{\partial \dot{z_{i}}}, Z_{1}, Z_{2}, \ldots, Z_{n} \in \mathcal{O}_{n, p} ; \text { y } \operatorname{m.c.d.}\left(Z_{1}, Z_{2}, \ldots, Z_{n}\right)=1
$$

en donde $\mathcal{O}_{n, p}$ es el anillo de gérmenes de las funciones analíticas en $p$. En lo sucesivo, denotaremos por $\mathcal{F}_{Z}$ a esta foliación, diremos que el campo $Z$ genera la foliación $\mathcal{F}_{Z}$ y las funciones $Z_{i}$ se llamarán componentes de $Z$. El lector interesado en conocer detalles sobre la teoría de funciones analíticas de varias complejas y la teoría de las variedades analíticas complejas, puede consultar [8] [9], [10], [11] y [13]. Sea $p \in \mathcal{M}^{n}$ y consideremos una carta $(U, \phi)$ de $\mathcal{M}^{n}$ alrededor del punto $p$ tal que $\phi(p)=0 \in \mathbb{C}^{n}$, claramente $Z_{i} \circ \phi^{-1}$ es una función analítica de varias variables complejas definida en una vecindad del origen y por lo tanto, ella tiene un desarrollo en series de potencias

$$
Z_{i} \circ \phi^{-1}=\sum_{k \geq 0} Z_{i}^{k}, \quad 1 \leq i \leq n
$$

\footnotetext{
${ }^{1}$ Universidad Nacional Mayor de San Marcos. Facultad de Ciencias Matemáticas.
} 
donde los $Z_{i}^{k}$ son polinomios homogéneos de grado $k$ en $n$ variables complejas. El orden de $Z_{i} \circ \phi^{-1}$ en el $0 \in \mathbb{C}^{n}$ es, por definición, el menor número entero $\nu_{i}$ tal que $Z_{i}^{k} \equiv 0$, para todo $k<\nu_{i}$ y $Z_{i}^{\nu_{i}} \not \equiv 0$. No es difícil probar que el número $\nu_{i}$ es independiente de la elección de la carta $(U, \phi)$, por esta razón el entero $\nu_{i}$ es llamado el orden de $Z_{i}$ en $p$ y lo denotamos por $\operatorname{ord}_{p}\left(Z_{i}\right)$. La multiplicidad algebraica de la foliación $\mathcal{F}_{Z}$ (o del campo $Z$ ) en el punto $p \in \mathcal{M}^{n}$, denotada por $m_{p}\left(\mathcal{F}_{Z}\right.$ ) (o simplemente por $m_{p}(Z)$ ), es definida como el mínimo de los órdenes $\operatorname{ord}_{p}\left(Z_{i}\right)$. Un punto $p \in \mathcal{M}^{n}$ es llamado punto singular de la foliación $\mathcal{F}_{Z}$ (o del campo $Z$ ) si y sólo si $m_{p}(Z) \geq 1$, en caso contrario decimos que $p$ es un punto regular. El conjunto de todos los puntos singulares de la foliación $\mathcal{F}_{Z}$ será denotado por $\operatorname{Sing}\left(\mathcal{F}_{Z}\right)$. Un punto $p \in \mathcal{M}^{n}$ es llamado singularidad aislada de $Z$ si y sólo si $p \in \operatorname{Sing}\left(\mathcal{F}_{Z}\right)$ y existe una vecindad abierta $U \subseteq \mathcal{M}^{n}$ de $p$ tal que todos los elementos de $U-\{p\}$ son puntos regulares de $Z$.

Sea $E: \tilde{\mathcal{M}}^{n} \rightarrow \mathcal{M}^{n}$ el blow-up centrado en el punto $p \in \operatorname{Sing}\left(\mathcal{F}_{Z}\right)$. Entonces existe una única manera de extender $E^{*}\left(\mathcal{F}_{Z}-\{p\}\right)$ a una foliación analítica singular $\tilde{\mathcal{F}}_{Z}$ sobre una vecindad del espacio proyectivo $\mathbb{C} P(n-1)=E^{-1}(p) \subset \tilde{\mathcal{M}}^{n}$, con conjunto singular de codimension mayor o igual que 2. En este. caso decimos que $\tilde{\mathcal{F}}_{Z}$ es el transformado estricto de $\mathcal{F}_{Z}$ por $E$. El lector interesado en mayores detalles sobre el tema, puede consultar las referencias [7], [12], [2] y [5].

Finalmente, decimos que $p$ es una singularidad no dicrítica de $\mathcal{F}_{Z}$ si y sólo si $E^{-1}(p)$ es invariante por $\tilde{\mathcal{F}}_{Z}$, es decir, $E^{-1}(p)$ es unión de hojas y singularidades de $\tilde{\mathcal{F}}_{Z}$. En caso contrario, $p$ es llamado singularidad dicrítica. El conjunto de las foliaciones analíticas por curvas sobre $\mathcal{M}^{n}$ con una singularidad dicrítica aislada, será denotado por $\mathcal{D}^{n}$.

\section{LA HIPERSUPERFICIE DE TANGENCIA}

Sea $\mathcal{M}^{n}$ una variedad analítica compleja de dimensión $n$ y consideremos en ella una foliación analítica singular por curvas $\mathcal{F}_{Z}$ en $\mathcal{M}^{n}$. Suponga que $p \in \mathcal{M}^{n}$ es una singularidad aislada de $\mathcal{F}_{Z}$. Sea $z=\left(z_{1}, \ldots, z_{n}\right)$ las coordenadas locales de una vecindad de $p$ en $\mathcal{M}^{n}$ tal que $p=(0, \ldots, 0) \in \mathbb{C}^{n}$. En estas coordenadas $\mathcal{F}_{Z}$ es generado por un campo vectorial holomorfo $Z=\sum_{i=1}^{n} Z_{i} \frac{\partial}{\partial z_{i}}$. If $m_{0}(Z)=\nu,\left(\nu \in \mathbb{Z}^{+}\right)$ entonces las componentes $Z_{i}$ de $Z$ tienen un desarrollo en serie de Taylor alrededor $\operatorname{del} 0 \in \mathbb{C}^{n}$

$$
Z_{i}=\sum_{k \geq \nu} Z_{k}^{i}, \quad 1 \leq i \leq n
$$

donde los $Z_{k}^{i}$ son polinomios homogéneos de grado $k$. 
Para cada $j=1, \ldots, n$ consideramos $U_{j}=\left\{\left(z_{1}, \ldots, z_{n}\right) \in \mathbb{C}^{n}: z_{j} \neq 0\right\}$ y $\tilde{U}_{j}=$ $E^{-1}\left[U_{j}\right]$, donde $E$ es el blowing-up centrado en $0 \in \mathbb{C}^{n}$. En $\tilde{U}_{j}$ introducimos las coordenadas $\left(y_{1}, \ldots, y_{n}\right)$, luego $E$ se expresa como:

$$
E\left(y_{1}, \ldots, y_{n}\right)=\left(z_{1}, \ldots, z_{n}\right) ; \text { donde } z_{i}=y_{i} y_{j} \text { si } i \neq j \text { y } z_{j}=y_{j}
$$

Además

$$
E^{-1}(0) \cap \tilde{U}_{j}=\left\{\left(y_{1}, \ldots, y_{n}\right) \in \tilde{U}_{j}: y_{j}=0\right\}
$$

En esta carta, el pull-back de $Z$ by $E$ es generado por:

$$
E^{*} Z=Z_{j} \circ E \frac{\partial}{\partial y_{j}}+\sum_{1 \leq i \neq j \leq n}\left(\frac{Z_{i} \circ E-y_{i} Z_{j} \circ E}{y_{j}}\right) \frac{\partial}{\partial y_{j}}
$$

de (2.1):

$$
Z_{i} \circ E(y)=\sum_{k \geq \nu} y_{j}^{k} A_{k}^{i}(\hat{y}), \text { con } y=\left(y_{1}, \ldots, y_{n}\right), \hat{y}=\left(y_{1}, \ldots, y_{j-1}, 1, y_{j+1}, \ldots, y_{n}\right)
$$

De (2.4):

$$
E^{*} Z(y)=\left(\sum_{k \geq \nu} y_{j}^{k} Z_{k}^{j}(\hat{y})\right) \frac{\partial}{\partial y_{j}}+\sum_{1 \leq i \neq j \leq n}\left(\sum_{k \geq \nu} y_{j}^{k-1}\left[Z_{k}^{i}(\hat{y})-y_{i} Z_{k}^{j}(\hat{y})\right]\right) \frac{\partial}{\partial y_{i}}
$$

En [1] se demuestra que, por ser $0 \in \mathbb{C}^{n}$ una singularidad dicrítica de $Z$, podemos dividir (2.5) por $y_{j}^{\nu}$ y definir $\tilde{Z}=\frac{E^{*} Z}{y_{j}^{\nu}}$. Se sigue que $\tilde{\mathcal{F}}_{Z}$ es la foliación generada por $\tilde{Z}$ en una vecindad de $E^{-1}(0) \cap \tilde{U}_{j}$, y de $(2.5)$ se tiene que:

$$
\tilde{Z}=\sum_{i=1}^{n} \tilde{Z}_{i} \frac{\partial}{\partial y_{i}}
$$

donde:

$$
\left\{\begin{array}{l}
\tilde{Z}_{i}\left(y_{1}, \ldots, y_{n}\right)=\sum_{k \geq \nu} y_{j}^{k-\nu}\left[Z_{k+1}^{i}(\hat{y})-y_{i} Z_{k+1}^{j}(\hat{y})\right], 1 \leq i \leq n, i \neq j \\
\tilde{Z}_{j}\left(y_{1}, \ldots, y_{n}\right)=P_{\nu-1}(\hat{y})+\sum_{k \geq \nu+1} y_{j}^{k-\nu} Z_{k}^{j}(\hat{y}) .
\end{array}\right.
$$

Luego es posible asociar a $J_{0}^{\nu}(Z)$, el primer jet no nulo de $\mathcal{F}_{Z}$ en 0 , un polinomio homogéneo $P_{\nu-1}$ de $n$ variables, de grado $\nu-1$ tal que:

$$
J_{p}^{\nu}(Z)=P_{\nu-1} R
$$

donde $R(z)=\sum_{i=1}^{n} z_{i} \frac{\partial}{\partial z_{i}}$ es el campo radial. 
El polinomio $P_{\nu-1}$ define una hipersuperficie algebraica $S$ sobre el espacio proyectivo $E^{-1}(0)=\mathbb{C} P(n-1)$ :

$$
S=\left\{\left[z_{1} ; \ldots ; z_{n}\right] \in \mathbb{C} P(n-1): P_{\nu-1}\left(z_{1}, \ldots, z_{n}\right)=0\right\}
$$

Observe que $S$ contiene todas las singularidades del transformado estricto $\tilde{\mathcal{F}}_{Z}$. La hipersuperficie $S$ tiene la siguiente interpretación geométrica: $\operatorname{Si} \tilde{p} \in S-\operatorname{Sing}\left(\tilde{\mathcal{F}}_{Z}\right)$ entonces la hoja $\tilde{L}$ de $\tilde{\mathcal{F}}_{Z}$ que pasa por $\tilde{p}$ es tangente al espacio proyectivo $E^{-1}(0)$ y si $\tilde{p} \in E^{-1}(0)-S$ entonces $\tilde{L}$ es transversal a $E^{-1}(0)$.

Un caso importante ocurre cuando $\operatorname{Sing}\left(\tilde{\mathcal{F}}_{Z}\right)=\emptyset$, es decir, cuando el transformado estricto de $\mathcal{F}_{Z}$ no tiene singularidades en $\tilde{\mathcal{M}}^{n}$. Denotaremos por $\mathcal{D}_{0}^{n}$ al conjunto de tales foliaciones. Observe que, en este caso, cada $\tilde{p} \in E^{-1}(0)$ es un punto regular de $\tilde{\mathcal{F}}_{Z}$. Por lo tanto, es posible definir el indice de intersección $i_{0}\left(E^{-1}(0), \tilde{L}\right)$ entre la hoja $\tilde{L}$ de $\tilde{\mathcal{F}}_{Z}$ que pasa por $\tilde{p} \in E^{-1}(0)$ y el espacio proyectivo $E^{-1}(0)$.

\section{EL ÍNDICE DE INTERSECCIÓN}

Sea $\mathcal{F}_{Z} \in \mathcal{D}_{0}^{n}$ generado por el campo vectorial holomorfo $Z$, sin pérdida de generalidad, podemos suponer que la singularidad aislada dicrítica es el $0 \in \mathbb{C}^{n}$. Sabemos que cualquier punto $\tilde{p} \in E^{-1}(0)=\mathbb{C} P(n-1)$ es un punto regular de la foliación $\tilde{\mathcal{F}}_{Z}$. Sea $\tilde{L}$ la hoja de $\tilde{\mathcal{F}}_{Z}$ que pasa por $\tilde{p}$. Desde que $E^{-1}(0)$ y $\tilde{L}$ son subvariedades analíticas de $\mathbb{C}^{n}$ de dimensiones complementarias (ver [6]) y $\tilde{p} \in E^{-1}(0) \cap \tilde{L}$, podemos definir el indice de intersección $i_{\tilde{p}}\left(E^{-1}(0), \tilde{L}\right)$ de la hoja $\tilde{L}$ con el espacio proyectivo $E^{-1}(0)$ en el punto $\tilde{p}$. En efecto, podemos suponer, sin pérdida de generalidad, que $\tilde{p}$ está en la carta de blow-up en donde $E$ se expresa como:

$$
E\left(y_{1}, \ldots, y_{n}\right)=\left(y_{1} y_{n}, \ldots, y_{n-1} y_{n}, y_{n}\right)=\left(z_{1}, \ldots, z_{n}\right)
$$

Desde que $\tilde{p}=\left(y_{1}^{0}, \ldots, y_{n-1}^{0}, 0\right)$ es un punto regular, la hoja $\tilde{L}$ puede ser localmente parametrizada por una función analítica

$$
\tilde{\alpha}=\left(\tilde{\alpha}_{1}, \ldots, \tilde{\alpha}_{n}\right):\left(\mathbb{D}_{\epsilon}, 0\right) \rightarrow(\tilde{L}, \tilde{p})
$$

donde $\mathbb{D}_{\epsilon}=\{T \in \mathbb{C}:|T|<\epsilon\}$, tal que

$$
\begin{aligned}
& \tilde{\alpha}^{\prime}(T)=\tilde{Z}(\tilde{\alpha}(T)), \quad \forall T \in \mathbb{D}_{\epsilon} \\
& \tilde{\alpha}(0)=\tilde{p} .
\end{aligned}
$$

Desde que $E^{-1}(0)=\left\{y_{n}=0\right\}$, podemos definir:

$$
i_{\tilde{p}}\left(E^{-1}(0), \tilde{L}\right)=\operatorname{ord}_{0}\left(\tilde{\alpha}_{n}\right)
$$


es decir, $i_{\tilde{p}}\left(E^{-1}(0), \tilde{L}\right)=m \Longleftrightarrow \tilde{\alpha}_{n}(T)=T^{m} \tilde{\varepsilon}_{n}(T)$ donde $\tilde{\varepsilon}$ es una función analítica y $\tilde{\varepsilon}_{n}(0) \neq 0$. Es claro que $i_{\tilde{p}}\left(E^{-1}(0), \tilde{L}\right) \geq 1, \forall \tilde{p} \in E^{-1}(0)$.

El índice de intersección puede ser geométricamente interpretado como el número de puntos de intersección de $\tilde{L}$ con una pequeña traslación del espacio proyectivo $\left\{y_{n}=\delta\right\}$, con $\delta \neq 0$. Para mayor información sobre las propiedades del índice de intersección entre subvariedades analíticas de dimensiones complementarias, el lector debe consultar [6].

Observe que si $\tilde{p} \in E^{-1}(0)$ entonces $\tilde{\alpha}_{n}^{\prime}(0)=P_{\nu-1}\left(y_{1}^{0}, \ldots, y_{n-1}^{0}, 1\right)$ entonces concluimos que $i_{\tilde{p}}\left(E^{-1}(0), \tilde{L}\right)=1$ si y sólo si $\tilde{p} \in E^{-1}(0)-S$ si y sólo si $\tilde{L}$ es transversal a $E^{-1}(0)$ en $\tilde{p}$.

En [3] se prueba que el índice de intersección es un invariante topoógico. En efecto, sean $\mathcal{F}_{Z}$ y $\mathcal{F}_{Z^{\prime}}$ dos elementos de $\mathcal{D}_{0}^{n}$ tales que $\mathcal{F}_{Z}$ y $\mathcal{F}_{Z^{\prime}}$ son topológicamente conjugados por un germen de homeomorfismo $h$ (i. e. si $L \in \mathcal{F}_{Z}$ entonces $h[L] \in \mathcal{F}_{Z^{\prime}}$ ). Sea $\tilde{p} \in E^{-1}(0)-S$, donde $S$ es la hipersuperficie de tangencia de $\mathcal{F}_{Z}$, desde que $\tilde{L}$, la hoja de $\tilde{\mathcal{F}}_{Z}$ que pasa por $\tilde{p}$, es transversal al espacio proyectivo $E^{-1}(0)$, tenemos que $i_{\tilde{p}}\left(E^{-1}(0), \tilde{L}\right)=1$. Consideremos $\tilde{U} \operatorname{vecindad}$ de $\tilde{p}$ tal que $\left.\tilde{L}\right|_{\tilde{U}} \cap E^{-1}(0)=\{\tilde{p}\}$ y $U$ vecindad de $0 \in \mathbb{C}^{n}$ tal que $E^{-1}[U-\{0\}] \subseteq \tilde{U}$. Si $L=\overrightarrow{E\left[\left.\tilde{L}\right|_{\tilde{U}}-\{\tilde{p}\}\right]}$ entonces $L-\{0\}$ es una hoja de $\mathcal{F}_{Z}$ y $L$ es una subvariedad analítica, irreducible de $U$, invariante por $Z$ y $\operatorname{dim}_{\mathbb{C}} L=1$.

De la definición de $h$, se sigue que $h[L-\{0\}]$ es una hoja de $\mathcal{F}_{Z^{\prime}}$. Sea $U^{\prime}=h[U]$ y $L^{\prime}=\overline{h[L-\{0\}]}$, se sigue del teorema de Remmert-Stein que $L^{\prime}$ es una subvariedad analítica, irreducible de $U^{\prime}$, con singularidad aislada en $0 \in \mathbb{C}^{n}$ y $\operatorname{dim}_{\mathbb{C}} L^{\prime}=1$. Desde que $L^{\prime}-\{0\} \in \mathcal{F}_{Z^{\prime}}$ entonces $E^{-1}[U-\{0\}] \in \mathcal{F}_{Z^{\prime}}$. Por lo tanto $\tilde{L}^{\prime}=\overline{E^{-1}[U-\{0\}]}$ esta contenido en una hoja de $\tilde{\mathcal{F}}_{Z^{\prime}}$ y $\tilde{L}^{\prime} \cap E^{-1}(0)=\left\{p^{\prime}\right\}$. Bajo estas condiciones, tenemos el siguiente resultado:

Teorema. (ver [3]) La multiplicidad algebraica de una foliación de $\mathcal{D}_{0}^{n}$ y el índice de intersección, son invariantes topológicos. Más específicamente, sean $\mathcal{F}_{Z}, \mathcal{F}_{Z^{\prime}} \in \mathcal{D}_{0}^{n}$, $\tilde{p}, \tilde{p}^{\prime}, \tilde{L}$ y $\tilde{L}^{\prime}$ como antes. Si $\mathcal{F}_{Z}$ y $\mathcal{F}_{Z^{\prime}}$ son topológicamente conjugados entonces

(i) $m_{0}(Z)=m_{0}\left(Z^{\prime}\right)$

(ii) $i_{\tilde{p}}\left(E^{-1}(0), \tilde{L}\right)=i_{\tilde{p}^{\prime}}\left(E^{-1}(0), \tilde{L}^{\prime}\right)$.

\section{EL ÍNDICE DE INTERSECCIÓN A LO LARGO DE UNA COMPONENTE IRREDUCIBLE DE $S$}

Sea $\mathcal{F}_{Z} \in \mathcal{D}_{0}^{n}$ la foliación generada por $Z=\sum_{i=1}^{n} Z_{i} \frac{\partial}{\partial z_{i}}$ con $Z_{i}=\sum_{k \geq \nu} Z_{k}^{i}$, en donde los $Z_{k}^{i}$ son polinomios homogéneos de grado $k$ y $m_{0}\left(\mathcal{F}_{Z}\right)=\nu$. $\operatorname{Sean} J_{0}^{\nu}(Z)=P_{\nu-1}$. 


$$
\begin{aligned}
& \left(\sum_{i=1}^{n} z_{i} \frac{\partial}{\partial z_{i}}\right) \text { el primer Jet no nulo de } Z \text { y } \\
& \qquad S=\left\{\left[z_{1} ; \ldots ; z_{n}\right] \in \mathbb{C} P(n-1): P_{\nu-1}\left(z_{1}, \ldots, z_{n}\right)=0\right\}
\end{aligned}
$$

la hipersuperficie de tangencia de $\mathcal{F}_{Z}$. Sabemos que la foliación $\tilde{\mathcal{F}}_{Z}$ es generada por el campo $\tilde{Z}$ el cual, en la carta $\tilde{U}_{n}$ of $\tilde{\mathbb{C}}^{n}$, se expresa como:

$$
\tilde{Z}=\sum_{i=1}^{n} \tilde{Z}_{i} \frac{\partial}{\partial y_{i}}
$$

donde:

$$
\left\{\begin{array}{l}
\tilde{Z}_{i}\left(y_{1}, \ldots, y_{n}\right)=\sum_{k \geq \nu} y_{n}^{k-\nu}\left[Z_{k+1}^{i}(\hat{y})-y_{i} Z_{k+1}^{n}(\hat{y})\right], 1 \leq i \leq n-1 \\
\tilde{Z}_{n}\left(y_{1}, \ldots, y_{n}\right)=P_{\nu-1}(\hat{y})+\sum_{k \geq \nu+1} y_{n}^{k-\nu} A_{k}^{n}(\hat{y}) .
\end{array}\right.
$$

y $\hat{y}=\left(y_{1}, \ldots, y_{n-1}, 1\right)$.

Dado $\tilde{p}=\left(y_{1}^{0}, \ldots, y_{n-1}^{0}, 0\right) \in E^{-1}(0) \cap \tilde{U}_{n}$, consideramos $\tilde{L}$ la hoja de $\tilde{\mathcal{F}}_{Z}$ que pasa por $\tilde{p}$, la cual puede ser parametrizada por la curva analítica:

donde

$$
\tilde{\alpha}=\left(\tilde{\alpha}_{1}, \ldots, \tilde{\alpha}_{n}\right):\left(\mathbb{D}_{\epsilon}, 0\right) \longrightarrow(\tilde{L}, \tilde{p})
$$

$$
\left\{\begin{array}{l}
\tilde{\alpha}^{\prime}(T)=\tilde{Z}(\tilde{\alpha}(T)), \text { i.e. } \tilde{\alpha}_{i}^{\prime}(T)=\tilde{Z}_{i}(\tilde{\alpha}(T)), \quad \forall 1 \leq i \leq n \\
\tilde{\alpha}(0)=\tilde{p} .
\end{array}\right.
$$

Por lo tanto $\tilde{\alpha}_{n}^{\prime}(0)=\tilde{A}_{n}\left(y_{1}^{0}, \ldots, y_{n-1}^{0}, 0\right)=P_{\nu-1}\left(y_{1}^{0}, \ldots, y_{n-1}^{0}, 1\right)$. Concluimos que:

$$
i_{\tilde{p}}\left(E^{-1}(0), \tilde{L}\right)>1 \Longleftrightarrow \tilde{p} \in S
$$

Dado $\tilde{p} \in S$, vamos a calcular $\tilde{\alpha}^{\prime \prime}(0)$. De (4.3): $\tilde{\alpha}_{n}^{\prime \prime}(T)=\sum_{i=1}^{n} \frac{\partial \tilde{Z}_{n}}{\partial y_{i}}(\tilde{\alpha}(T)) \tilde{\alpha}_{i}^{\prime}(T)$, luego

$$
\begin{aligned}
\tilde{\alpha}_{n}^{\prime \prime}(0) & =\sum_{i=1}^{n-1} \frac{\partial \tilde{Z}_{n}}{\partial y_{i}}(\tilde{p}) \tilde{Z}_{i}(\tilde{p}) \\
& =\sum_{i=1}^{n-1} \frac{\partial \tilde{P}_{\nu-1}}{\partial z_{i}}\left(\tilde{y}_{0}\right)\left[Z_{\nu+1}^{i}\left(\tilde{y}_{0}\right)-y_{i}^{0} Z_{\nu+1}^{n}\left(\tilde{y}_{0}\right)\right] \\
& =\sum_{i=1}^{n-1} \frac{\partial \tilde{P}_{\nu-1}}{\partial z_{i}}\left(\tilde{y}_{0}\right) Z_{\nu+1}^{i}\left(\tilde{y}_{0}\right)-Z_{\nu+1}^{n}\left(\tilde{y}_{0}\right) \sum_{i=1}^{n-1} y_{i}^{0} \frac{\partial \tilde{P}_{\nu-1}}{\partial z_{i}}\left(\tilde{y}_{0}\right)
\end{aligned}
$$


donde $\tilde{y}_{0}=\left(y_{1}^{0}, \ldots, y_{n-1}^{0}, 1\right)$. Por lo tanto:

$$
\tilde{\alpha}_{n}^{\prime \prime}(0)=\sum_{i=1}^{n} \frac{\partial \tilde{P}_{\nu-1}}{\partial z_{i}}\left(y_{1}^{0}, \ldots, y_{n-1}^{0}, 1\right) Z_{\nu+1}^{i}\left(y_{1}^{0}, \ldots, y_{n-1}^{0}, 1\right) .
$$

Definimos el polinomio homogéneo $Q_{2 \nu-1}$ de grado $2 \nu-1$ como:

$$
Q_{2 \nu-1}=d P_{\nu-1}\left[Z_{\nu+1}\right]=\sum_{i=1}^{n} \frac{\partial P_{\nu-1}}{\partial z_{i}} Z_{\nu+1}^{i}
$$

y denotamos por $S^{*}$ a la hipersuperficie algebraica generada por $Q_{2 \nu-1}$, i.e.

$$
S^{*}=\left\{\left[z_{1} ; \ldots ; z_{n}\right] \in \mathbb{C} P(n-1): Q_{2 \nu-1}\left(z_{1}, \ldots, z_{n}\right)=0\right\}
$$

de (4.4) deducimos que:

$$
i_{\tilde{p}}\left(E^{-1}(0), \tilde{L}\right)>2 \Longleftrightarrow \tilde{p} \in S \cap S^{*} .
$$

Note que los puntos singulares de la hipersuperficie de tangencia $S$ are contained in $S^{*}$, sin embargo $S \cap S^{*}$ contiene otros puntos $\tilde{p}$ los cuales no son singularidades de $S$ pero $i_{\tilde{p}}\left(E^{-1}(0), \tilde{L}\right)>2$.

Ejemplo.- Considere el campo vectorial holomorfo

$$
Z\left(z_{1}, z_{2}, z_{3}\right)=\left(z_{1} z_{3}, z_{2} z_{3}+z_{1}^{3}, z_{3}^{2}+z_{1}^{2} z_{2}-z_{2}^{3}\right)
$$

Claramente $\mathcal{F}_{Z} \in \mathcal{D}_{0}^{3}, m_{0}(Z)=2$ y el polinomio homogéneo de grado 1 asociado a $Z$ es $P_{1}\left(z_{1}, z_{2}, z_{3}\right)=z_{3}$. Entonces, la hipersuperficie de tangencia $S$ of $\mathcal{F}_{Z}$ viene dada por:

$$
S=\left\{\left[z_{1} ; z_{2} ; z_{3}\right] \in \mathbb{C} P(2): z_{3}=0\right\}
$$

En la carta $\tilde{U}_{1}$ de $\tilde{\mathbb{C}}^{3}$ en donde el blowing-up es expresado por $E\left(y_{1}, y_{2}, y_{3}\right)=$ $\left(y_{1}, y_{1} y_{2}, y_{1} y_{3}\right)=\left(z_{1}, z_{2}, z_{3}\right)$, tenemos que:

$$
\tilde{Z}\left(y_{1}, y_{2}, y_{3}\right)=\left(y_{3}, 1, y_{2}-y_{2}^{3}\right)
$$

Sea $\tilde{p}=\left(0, y_{2}^{0}, 0\right) \in S$ and $\tilde{L} \in \tilde{\mathcal{F}}_{Z}$ la hoja que pasa por $\tilde{p}$. No es difícil ver que $\tilde{L}$ esta localmente parametrizado por $\tilde{\alpha}(T)=\left(\tilde{\alpha}_{1}(T), \tilde{\alpha}_{2}(T), \tilde{\alpha}_{3}(T)\right)$, donde:

$$
\left\{\begin{array}{l}
\tilde{\alpha}_{1}(T)=\frac{y_{2}^{0}-\left(y_{2}^{0}\right)^{3}}{2} T^{2}+\frac{1-3\left(y_{2}^{0}\right)^{2}}{6} T^{3}-\frac{1}{4} y_{2}^{0} T^{4}-\frac{1}{20} T^{5} \\
\tilde{\alpha}_{2}(\dot{T})=T+y_{2}^{0} \\
\tilde{\alpha}_{3}(T)=\left[y_{2}^{0}-\left(y_{2}^{0}\right)^{3}\right] T+\frac{1-3\left(y_{2}^{0}\right)^{2}}{2} T^{2}-y_{2}^{0} T^{3}-\frac{1}{4} T^{4}
\end{array}\right.
$$

luego: 
Concluimos que:

$$
\tilde{\alpha}_{1}(T)=\left\{\begin{array}{l}
T^{2} \tilde{\xi}_{1}(T), \text { con } \tilde{\xi}_{1}(0) \neq 0 ; \text { si } y_{2}^{0} \neq 0,-1,1 \\
T^{3} \tilde{\xi}_{1}(T), \text { con } \tilde{\xi}_{1}(0) \neq 0 ; \text { si } y_{2}^{0}=0,-1,1
\end{array}\right.
$$

$$
i_{\tilde{p}}\left(E^{-1}(0), \tilde{L}\right)= \begin{cases}2, & \text { si } \tilde{p} \notin\{[1 ; 1 ; 0],[1 ;-1 ; 0],[1 ; 0 ; 0]\} \\ 3, & \text { en otro caso. }\end{cases}
$$

De otro lado $Q_{3}\left(z_{1}, z_{2}, z_{3}\right)=d P_{1}\left[Z_{3}\right]\left(z_{1}, z_{2}, z_{3}\right)=z_{1}^{2} z_{2}-z_{2}^{3}$, luego:

$$
S^{*}=\left\{\left[z_{1} ; z_{2} ; z_{3}\right] \in \mathbb{C} P(2): z_{1}^{2} z_{2}-z_{2}^{3}=0\right\}
$$

$\mathrm{y}$

$$
S \cap S^{*}=\{[1 ; 1 ; 0],[1 ;-1 ; 0],[1 ; 0 ; 0]\} .
$$

Por lo tanto, en los tres puntos de $S \cap S^{*}$ el índice de intersección es 3 y si $\tilde{p} \in S-S^{*}$, entonces $i_{\tilde{p}}\left(E^{-1}(0), \tilde{L}\right)=2$.

El ejemplo anterior nos muestra que el índice de intersección es constante en casi todo punto de cada componente irreducible de la hipersuperficie de tangencia $S$. Más específicamente, sea $\mathcal{F}_{Z} \in \mathcal{D}_{0}^{n}$ con $m_{0}\left(\mathcal{F}_{Z}\right)=\nu$ tal que $J_{0}^{\nu}(Z)=P_{\nu-1}\left(\sum_{i=1}^{n} z_{i} \frac{\partial}{\partial z_{i}}\right)$. Desde que $P_{\nu-1}$ es un polinomio homogéneo de $n$ variables, puede ser factorizado:

$$
P_{\nu-1}=F_{1}^{r_{1}} \cdots F_{l}^{r_{l}}
$$

donde $\operatorname{los} F_{j}(1 \leq j \leq l)$ son polinomios homogéneos irreducibles de grado $\operatorname{deg}\left(F_{j}\right)=$ $g_{j}$. Esta factorización es única salvo factores constantes no nulos. De acuerdo a la factorización (4.8), podemos escribir la hipersuperficie de tangencia

$$
S=\left\{\left[z_{1} ; \ldots ; z_{n}\right] \in \mathbb{C} P(n-1): P_{\nu-1}\left(z_{1}, \ldots, z_{n}\right)=0\right\}
$$

como:

$$
S=r_{1} S_{1}+\cdots+r_{l} S_{l}
$$

donde:

$$
S_{j}=\left\{\left[z_{1} ; \ldots ; z_{n}\right] \in \mathbb{C} P(n-1): F_{j}\left(z_{1}, \ldots, z_{n}\right)=0\right\}(1 \leq j \leq l) .
$$

Las hipersuperficies algebraicas $S_{j}$ son llamadas las componentes irreducibles de $S$, mientras que los números enteros positivos $g_{j}$ y $r_{j}$ son llamados respectivamente el grado y la multiplicidad de $S_{j}$ in $S$. Es claro que el grado y la multiplicidad de $S_{j}$ in $S$ satisfacen la ecuación:

$$
\sum_{j=1}^{l} r_{j} g_{j}=\nu-1
$$

De manera análoga a (4.5), definimos los polinomios homogéneos: 


$$
G_{j}=d F_{j}\left[Z_{\nu+1}\right]=\sum_{i=1}^{n} \frac{\partial F_{j}}{\partial z_{i}} Z_{\nu+1}^{i}
$$

y denotamos por $S_{j}^{*}$ a la hipersuperficie algebraica:

$$
S_{j}^{*}=\left\{\left[z_{1} ; \ldots ; z_{n}\right] \in \mathbb{C} P(n-1): G_{j}\left(z_{1}, \ldots, z_{n}\right)=0\right\}(1 \leq j \leq l) .
$$

Note que los puntos singulares de la hipersuperficie $S_{j}$ están contenidos en $S_{j}^{*}$. Estamos listos para enunciar y demostrar nuestro principal resultado:

Teorema. Con las notaciones anteriores, sea $\tilde{p} \in S_{j}-S_{j}^{*}$ tal que $\tilde{p} \notin S_{i}, 1 \leq i \leq$ $l, i \neq j$. Entonces $i_{\tilde{p}}\left(E^{-1}(0), \tilde{L}\right)=r_{j}+1$

Demostración. Considérese $i \leq j \leq l$ ( $j$ fijo) y denotemos:

$$
P_{\nu-1}=F_{j}^{r_{j}} P \text { donde } P=F_{1}^{r_{1}} \cdots F_{j-1}^{r_{j-1}} F_{j+1}^{r_{j+1}} \cdots F_{l}^{r_{l}}
$$

Podemos suponer, sin pérdida de generalidad, que $\tilde{p}=\left(y_{1}^{0}, \ldots, y_{n-1}^{0}, 0\right) \in \tilde{U}_{n}$. El Teorema es consecuencia de la siguiente fórmula:

$$
\begin{aligned}
\tilde{\alpha}_{n}^{(k)}(T)= & \frac{r_{j} !}{\left(r_{j}-k+1\right) !} F_{j}^{r_{j}-k+1}(\hat{\alpha}(T)) P(\hat{\alpha}(T))^{\cdot}\left[\sum_{i=1}^{n-1} \tilde{\alpha}_{i}^{\prime}(T) \frac{\partial F_{j}}{\partial z_{i}}(\hat{\alpha}(T))\right]^{k-1}+ \\
& +\sum_{i=0}^{k-2} F_{j}^{r-i}(\hat{\alpha}(T)) H_{k, i}(T)+\sum_{i=0}^{k-1} \tilde{\alpha}_{n}^{(i)}(T) \psi_{k, i}(T)
\end{aligned}
$$

donde $2 \leq k \leq r_{j}+1, \hat{\alpha}(T)=\left(\tilde{\alpha}_{1}(T), \ldots, \tilde{\alpha}_{n-1}(T), 1\right)$ y $H_{k, i}, \psi_{k, i}$ son funciones analíticas de la variable $T$. En efecto, sabemos que $\tilde{\alpha}_{n}(0)=\tilde{\alpha}_{n}^{\prime}(0)=0$. Por inducción, suponga que $\tilde{\alpha}_{n}^{(i)}(0)=0, \forall 1 \leq i \leq r_{j}-1$ entonces:

$$
\begin{aligned}
\tilde{\alpha}_{n}^{\left(r_{j}\right)}(0)= & r_{j} ! F_{j}\left(\hat{y}_{0}\right) P\left(\hat{y}_{0}\right)\left[\sum_{i=1}^{n-1} \tilde{\alpha}_{i}^{\prime}(0) \frac{\partial F_{j}}{\partial z_{i}}\left(\hat{y}_{0}\right)\right]^{r_{j}-1}+ \\
& +\sum_{i=0}^{r_{j}-2} F_{j}^{r_{j}-i}\left(\hat{y}_{0}\right) H_{r_{j}, i}(0)+\sum_{i=0}^{r_{j}-1} \tilde{\alpha}_{n}^{(i)}(0) \psi_{r_{j}, i}(0)
\end{aligned}
$$

donde $\hat{y}_{0}=\left(y_{1}^{0}, \ldots, y_{n-1}^{0}, 1\right)$. Por lo tanto, $\tilde{\alpha}_{n}^{\left(r_{j}\right)}(0)=0$. De otro lado:

$$
\begin{aligned}
\tilde{\alpha}_{n}^{\left(r_{j}+1\right)}(0)= & r_{j} ! P\left(\hat{y}_{0}\right)\left[\sum_{i=1}^{n-1} \tilde{\alpha}_{i}^{\prime}(0) \frac{\partial F_{j}}{\partial z_{i}}\left(\hat{y}_{0}\right)\right]^{r_{j}}+\sum_{i=0}^{r_{j}-1} F_{j}^{r_{j}-i}\left(\hat{y}_{0}\right) H_{r_{j}+1, i}(0)+ \\
& +\sum_{i=0}^{r_{j}} \tilde{\alpha}_{n}^{(i)}(0) \psi_{r_{j}+1, i}(0)
\end{aligned}
$$


De (4.2) y desde que $\tilde{p} \in S_{j}$, tenemos:

$$
\begin{aligned}
\tilde{\alpha}_{n}^{\left(r_{j}+1\right)}(0) & =r_{j} ! P\left(\hat{y}_{0}\right)\left[\sum_{i=1}^{n-1}\left(Z_{\nu+1}^{i}\left(\hat{y}_{0}\right)-y_{i}^{0} Z_{\nu+1}^{n}\left(\hat{y}_{0}\right) \frac{\partial F_{j}}{\partial z_{i}}\left(\hat{y}_{0}\right)\right)\right]^{r_{j}} \\
& =r_{j} ! P\left(\hat{y}_{0}\right)\left[\sum_{i=1}^{n-1} Z_{\nu+1}^{i}\left(\hat{y}_{0}\right) \frac{\partial F_{j}}{\partial z_{i}}\left(\hat{y}_{0}\right)-Z_{\nu+1}^{n}\left(\hat{y}_{0}\right) \sum_{i=1}^{n-1} y_{i}^{0} \frac{\partial F_{j}}{\partial z_{i}}\left(\hat{y}_{0}\right)\right]^{r_{j}} \\
& =r_{j} ! P\left(\hat{y}_{0}\right)\left[\sum_{i=1}^{n} Z_{\nu+1}^{i}\left(\hat{y}_{0}\right) \frac{\partial F}{\partial z_{i}}\left(\hat{y}_{0}\right)\right]^{r_{j}}
\end{aligned}
$$

Por lo tanto $\tilde{\alpha}_{n}^{\left(r_{j}+1\right)}(0)=r_{j} ! P\left(y_{1}^{0}, \ldots, y_{n-1}^{0}, 1\right) \cdot G_{j}^{r_{j}}\left(y_{1}^{0}, \ldots, y_{n-1}^{0}, 1\right)$.

Desde que $\tilde{p} \notin S_{i}, \forall 1 \leq i \leq n, \quad i \neq j$, tenemos que $P\left(y_{1}^{0}, \ldots, y_{n-1}^{0}, 1\right) \neq 0$. Más aún $G_{j}\left(y_{1}^{0}, \ldots, y_{n-1}^{0}, 1\right) \neq 0$ (recuerde que $\left.\tilde{p} \notin S_{j}^{*}\right)$. Luego $\tilde{\alpha}_{n}^{\left(r_{j}+1\right)}(0) \neq 0$ y la demostración del Teorema esta lista.

Prueba de la fórmula (4.15): Procediendo por inducción sobre $k$, para $k=2$ la fórmula se cumple trivialmente. Suponiendo que es válida para $k$, debemos probarla para $k+1$ :

$$
\begin{aligned}
\tilde{\alpha}_{n}^{(k+1)}(T)= & \frac{r_{j} !}{\left(r_{j}-k\right) !} F_{j}^{r_{j}-k}(\hat{\alpha}(T)) P(\hat{\alpha}(T))\left[\sum_{i=1}^{n-1} \tilde{\alpha}_{i}^{\prime}(T) \frac{\partial F_{j}}{\partial z_{i}}(\hat{\alpha}(T))\right]^{k}+ \\
& +\frac{r_{j} !}{\left(r_{j}-k+1\right) !} F_{j}^{r_{j}-k+1}(\hat{\alpha}(T)) \frac{d}{d T}\left[P(\hat{\alpha}(T))\left(\sum_{i=1}^{n-1} \tilde{\alpha}_{i}^{\prime}(T) \frac{\partial F_{j}}{\partial z_{i}}(\hat{\alpha}(T))\right)^{r_{j}}\right] \\
& +\sum_{i=0}^{k-2}\left(r_{j}-i\right) F_{j}^{r_{j}-i-1}(\hat{\alpha}(T)) H_{k, i}(T) \frac{d}{d T}\left(F_{j}^{r_{j}-k}(\hat{\alpha}(T))\right)+ \\
& +\sum_{i=0}^{k-2} F_{j}^{r_{j}-i}(\hat{\alpha}(T)) H_{k, i}^{\prime}(T)+\sum_{i=0}^{k-1} \tilde{\alpha}_{n}^{(i+1)}(T) \psi_{k, i}(T)+\sum_{i=0}^{k-1} \tilde{\alpha}_{n}^{(i)}(T) \psi_{k, i}^{\prime}(T)
\end{aligned}
$$

Definimos:

$$
\begin{aligned}
H_{k+1, k-1}(T)= & \frac{r_{j} !}{\left(r_{j}-k+1\right) !} \frac{d}{d T}\left[P(\hat{\alpha}(T))\left(\sum_{i=1}^{n-1} \tilde{\alpha}_{i}^{\prime}(T) \frac{\partial F_{j}}{\partial z_{i}}(\hat{\alpha}(T))\right)^{r_{j}}\right] \\
& +\left(r_{j}-k+2\right) H_{k, k-2}(T) \frac{d}{d T}\left(F_{j}(\hat{\alpha}(T))\right)
\end{aligned}
$$




$$
\begin{aligned}
H_{k+1, i}(T) & =\left(r_{j}-i+1\right) H_{k, i-1}(T) \frac{d}{d T}\left(F_{j}(\hat{\alpha}(T))\right)+H_{k, i}^{\prime}(T) ; \quad 1 \leq i \leq k-2 \\
H_{k+1,0}(T) & =H_{k, 0}^{\prime}(T) \\
\psi_{k+1, k}(T) & =\psi_{k, k}(T) \\
\psi_{k+1, i}(T) & =\psi_{k, i-1}(T)+\psi_{k, i}^{\prime}(T) ; \quad 1 \leq i \leq k-1 \\
\psi_{k+1,0}(T) & =\psi_{k, 0}^{\prime}(T)
\end{aligned}
$$

Luego, tenemos que:

$$
\begin{aligned}
\tilde{\alpha}_{n}^{(k+1)}(T)= & \frac{r_{j} !}{\left(r_{j}-k\right) !} F_{j}^{r_{j}-k+1}(\hat{\alpha}(T)) P(\hat{\alpha}(T))\left[\sum_{i=1}^{n-1} \tilde{\alpha}_{i}^{\prime}(T) \frac{\partial F_{j}}{\partial z_{i}}(\hat{\alpha}(T))\right]^{k}+ \\
& +\sum_{i=0}^{k-1} F_{j}^{r_{j}-i}(\hat{\alpha}(T)) H_{k+1, i}(T)+\sum_{i=0}^{k} \tilde{\alpha}_{n}^{i}(T) \psi_{k+1, i}(T) .
\end{aligned}
$$

Por lo tanto, la fórmula esta probada.

\section{BIBLIOGRAFÍA}

[1] R. Benazic, Caracterización de singularidades dicríticas en foliaciones de dimension uno, PESQUIMAT, Vol. 1, N 1, (1998), p. 73-81.

[2] R. Benazic, Sobre el Número de Milnor, PESQUIMAT, Vol. III, N 1, (2000),p. 1-15.

[3] R. Benazic, Invarianza Topológica del índice de intersección, PESQUIMAT, Vol. IV, N 1, (2001),p. 31-38.

[4] C. Camacho, L. Neto, P. Sad, Topological Invariants and Equidesingularization for Holomorphic Vector Fields, J. Differential Geometry 20, (1984), p. 143-174.

[5] D. Cerveau, J. Mattei, Formes Intégrables Holomorphes Singulières, Asterisque, 97, (1982).

[6] E. Chirka, Complex Analytic Sets, MIA, Kluwer Academic Publishers. Dordrecht, Boston, London (1989).

[7] P. Griffiths, J. Harris, Principles of Algebraic Geometry, Wiley-Intersciencie, New York, (1978).

[8] R. Gunning, H. Rossi, Analytic functions of several complex variables, Prentice-Hall, Englewood Ćliffs, NJ., (1965).

[9] R. Gunning, Introduction to Holomorphic Functions Several Variables, Vol. I: Function Theory Wadsworth \& Brooks/Cole, 1990.

10] R. Gunning, Introduction to Holomorphic Functions Several Variables, Vol. II: Local Theory, Wadsworth \& Brooks/Cole, 1990.

[11] R. Gunning, Introduction to Holomorphic Functions Several Variables, Vol. III: Homological Theory, Wadsworth \& Brooks/Cole, 1990.

[12] J. Mattei, R. Moussu, Holonomie et intégrales premières, Ann. Sci. Ecole. Norm. Sup. (4) 13 (1980) p. 469-523.

[13] H. Whitney, Complex Analytic Varieties, Addison-Wesley Publishing Company, (1972). 